\title{
Functional Requirements-Based Automated Testing for Avionics
}

\author{
Youcheng Sun Martin Brain Daniel Kroening \\ University of Oxford, UK \\ Andrew Hawthorn Thomas Wilson Florian Schanda \\ Altran, UK \\ Francisco Javier Guzmán Jiménez Simon Daniel \\ Rolls-Royce, UK \\ Chris Bryan Ian Broster \\ Rapita Systems, UK
}

\begin{abstract}
We propose and demonstrate a method for the reduction of testing effort in safety-critical software development using DO-178 guidance. We achieve this through the application of Bounded Model Checking (BMC) to formal low-level requirements, in order to generate tests automatically that are good enough to replace existing labor-intensive test writing procedures while maintaining independence from implementation artefacts. Given that existing manual processes are often empirical and subjective, we begin by formally defining a metric, which extends recognized best practice from code coverage analysis strategies to generate tests that adequately cover the requirements. We then formulate the automated test generation procedure and apply its prototype in case studies with industrial partners. In review, the method developed here is demonstrated to significantly reduce the human effort for the qualification of software products under DO-178 guidance.
\end{abstract}

\section{Introduction}

DO-178C/ED-12C 1 provides guidance for the production of software for airbone systems. For each process of the life-cycle it lists the objectives for the life-cycle, the activities required to meet those objectives and explains what evidence is required to demonstrate that the objectives have been fulfilled. DO- $178 \mathrm{C}$ is the most recent version of the DO-178 family. DO-178 is thorough, and when the highest level is chosen, aids the production of highly reliable software. 
Developing to this guidance can be hard and time consuming. We will use one small aspect of this process as exemplar: the generation and verification of low-level tests against low-level requirements (LLRs) as described in the Software Reviews and Analyses part (Section 6 of the DO-178C). The objectives related to this particular area are: compliance with the low-level requirements (6.3.4.a), compliance with the software architecture (6.3.4.b), verifiability (6.3.4.c), conformance to standards (6.3.4.d), traceability (6.3.4.e), accuracy and consistency (6.3.4.f), the executable object code complies with the low-level requirements (6.4.c), the executable object code is robust with the low-level requirements (6.4.d), the executable object code is compatible with the target computer (6.4.e), normal range test cases (4 additional objectives, 6.4.2.1), robustness test cases (6 additional objectives, 6.4.2.2) and test coverage analysis (4 additional objectives, 6.4.4). A typical DO-178C test coverage analysis has two steps:

1. The first step is analysis of the coverage of requirements.

2. The second step is structural coverage analysis on the implementation.

The requirements-based coverage analysis establishes whether the software requirements are covered adequately, which may prompt a revision of the requirements-based tests. Subsequently, structural coverage analysis is applied to determine which part of the code is exercised by the requirements-based tests. Inadequate structural coverage in the second step indicates that requirements are missing or that implementation behaviour is unspecified. To this end, requirements-based testing derives a suite of tests from software requirements only, and must not use internal structure of the implementation.

Structural Coverage Analysis The most common form of structural coverage analysis is code coverage analysis, which measures the degree to which the source code of a program has been covered during execution. Criteria for code coverage include statement coverage (checking whether each statement in the program has been executed), branch coverage (checking whether each branch of conditional structures in the code has been taken), and Modified Condition/Decision coverage (MC/DC). In MC/DC analysis, a boolean decision consists of multiple boolean conditions such that every condition shall be evaluated to true and false and it is required that this switch changes the outcome of the final decision. DO-178C guidance requires $\mathrm{MC} / \mathrm{DC}$ coverage of function bodies.

Automating functional requirements-based testing Common requirements-based testing techniques [2] include equivalence partitioning, boundary value analysis, decision tables, and state transition testing. Equivalence partitioning and boundary value analysis are most relevant to the coverage criterion we propose. Equivalence class partitioning is a software testing technique that partitions each of the inputs (or outputs) to the unit under test into a finite number of disjoint equivalence classes. It can also be applied to the inputs to a conditional statement. It is usually used in conjunction with boundary value 
analysis. Boundary value analysis is a technique that generates test cases that exercise an input or predicate (conditional) variable just below, on, and just above the limits of valid ranges (equivalence partitions), the rationale being that errors tend to occur near, or on the partition boundaries.

The key goal of this paper is the automated creation of a functional test suite from formal, low-level requirements. Software requirements are usually written in natural language and for our approach these must then be translated into a formal specification in the form of pre- and post-conditions. The preconditions capture the calling context required before the function call, and the post-conditions capture the state of the system required after the function call.

There is a variety of approaches to automating specification-based testing. In particular, 3] is among first that applied Model Checking to generate tests from requirements specifications. For each condition tested, a trap property that violates this condition is generated and instrumented into the code: if a trap property is satisfied by the model checking, it means that the corresponding condition will be never met by the program; otherwise, a counterexample will be returned by the Model Checker that leads the program to the condition tested and a test vector can be thus derived. However, the method in [3] needs to call the function body in a black box fashion. In [4, structural coverage is applied to function specifications to generate testing conditions, which are then automatically matched with tests that already exist. 5] summarizes test selection strategies when using formal specifications to support testing. In such cases, post-conditions are only used to determine the outcome of the tests that are generated. Moreover, work like [6] and [7] test specifications that include temporal properties; though beyond the scope of the work in this paper, they establish interesting future directions.

\section{Main contributions.}

- We formally define a coverage criterion for generating testing conditions from software requirements. It is based on "MC/DC plus boundary analysis", extended with control flow coverage.

- We design an automated procedure for the test generation from pre- and post-conditions using a Model Checker.

- We implemented the procedure in Model Checking tool CBMC 88 and integrated it with RapiTestFramework (published by Rapita Systems). The resultant toolchain specializes in automatic testing under DO-178C and was applied in real-world case studies using source code for an avionics system provided by Rolls-Royce. We report here outcome from the case study work and experience learned from this project.

Structure of the paper In Section 2, we introduce the application context for our automatic test case generation approach. Section 3 gives the formal definition for functional requirements, in form of pre- and post-conditions. In 
Section 4 a coverage criterion is formulated for coverage analysis of requirements. Testing conditions generated according to the proposed criterion, which are then utilized by the automated testing framework developed in Section 5 to generated test cases. The method introduced so far has been implemented and integrated in a toolchain that is examined by two industrial case studies, and this is reported in Section 6. Finally, we conclude the paper and discuss future exploitations in Section 7.

\section{The Problem Formulation}

The work in this paper targets the scenario depicted in Figure 1, with our focus on using automated low-level requirements (LLRs) testing to minimize human effort. Within such a scheme, after high-level requirements (HLRs) are (mostly) manually interpreted as low-level requirements (LLRs), the function implementation (Impl) and the requirements testing are two independent procedures. The function implementation can be either manually written by software programmers or automatically generated by source code generation tools as in many model-based development platforms. In the end, test cases generated from LLRs must be validated against the function implementation (e.g., by code coverage level measurement), which are supplied as evidence for fulfilling DO-178C guidance.

Figure 1 depicts a suitable scheme for developing avionics software under DO-178C. The work in this paper specializes in the LLRs testing, which is nowadays performed manually by experienced engineers. We aim to automating this procedure, that is, automatically creating test cases for LLRs.

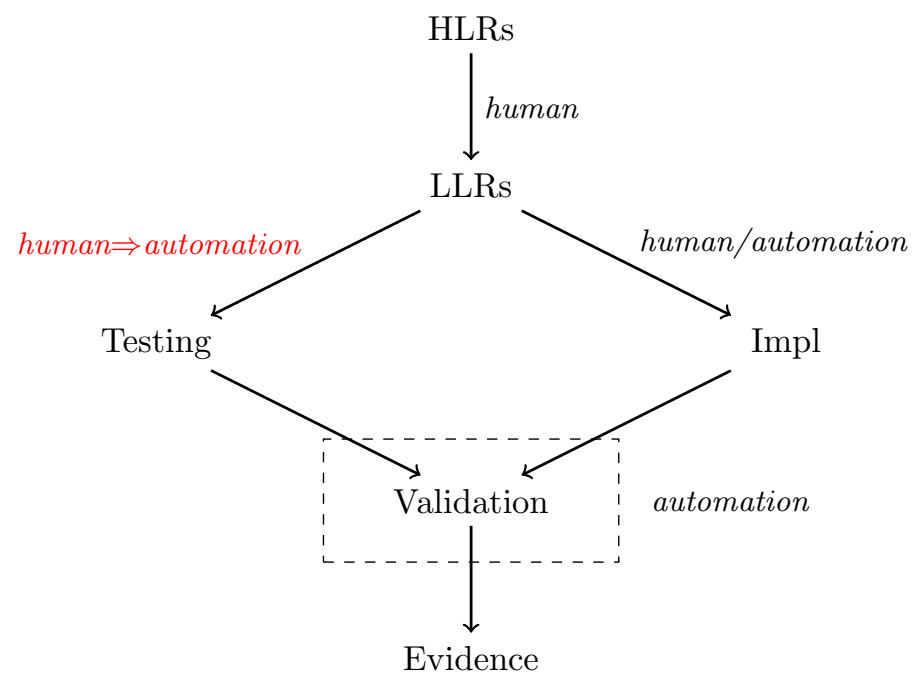

Figure 1: The targeted software development scheme 
Given the authors' experience in the field, the cost of developing tests against low-level requirements (as required by the objectives in DO-178C) equates to, on average, two days per procedure/function. This takes account of initial test development, test debugging and review. For a project with 250,000 lines of code and assuming an average size of 25 lines per procedure/function, this equates to 50 person-years of effort! Given this level of effort and expense, coupled with the generally held view that low-level testing is of relatively low value, there is a strong commercial incentive to consider ways of automating this aspect of development. The DO-333 Formal Methods Supplement 9] to RTCA DO-178C provides an approach to automating low-level verification through the use of proof, but few businesses are considering this option because of the perceived cost of using formal methods. This is because verification results from a formal methods tool (e.g., a Model Checker) can be used under DO-178C and DO-333 guidance, only if that tool has been certified or the verification process has been instrumented to generate the evidence. Either way is challenging.

The research described in this paper has used formal methods in another way: instead of mathematically proving the software, we use proof to generate tests that cover low-level verification objectives (listed earlier) through execution. We expect (at least) the following advantages of this approach.

- Easy adoption The automated tests represent a like-to-like replacement for existing (manual) test-writing procedures. Generated tests can be reviewed in the same way as those written by a skilled engineer without a background in formal methods.

- Consistency The usage of qualified tools that make use of formal methods improves consistency and completeness of testing by removing human variability.

- Improved development lifecycle An automated process encourages developers to specify requirements at the beginning of the development lifecycle and to adopt test-driven practices.

- Reduced cost As previously stated, a large percentage of the cost of safetycritical development is in low-level testing. Significant cost savings will be achieved by reducing or eliminating manual testing effort.

- Painless maintenance If the code under test is changed, a revised set of tests can be generated without additional effort.

- Extensibility When required, manual tests can be added alongside to complement generated tests in an identical format.

\section{The Requirement Specification}

The software requirements must be formally specified in order to perform the automatic test generation. Works on specification languages include 10 for C, 
[11] for the .NET, and SPARK Ada [12. Hierons et al. [5] provide a survey on formal specification techniques for testing.

Instead of digging into the details of some specific programming language syntax, also for both the convenience and generality, we assume that every requirement is specified as a Boolean expression bool_expr, defined following the mini syntax in Listing (1).

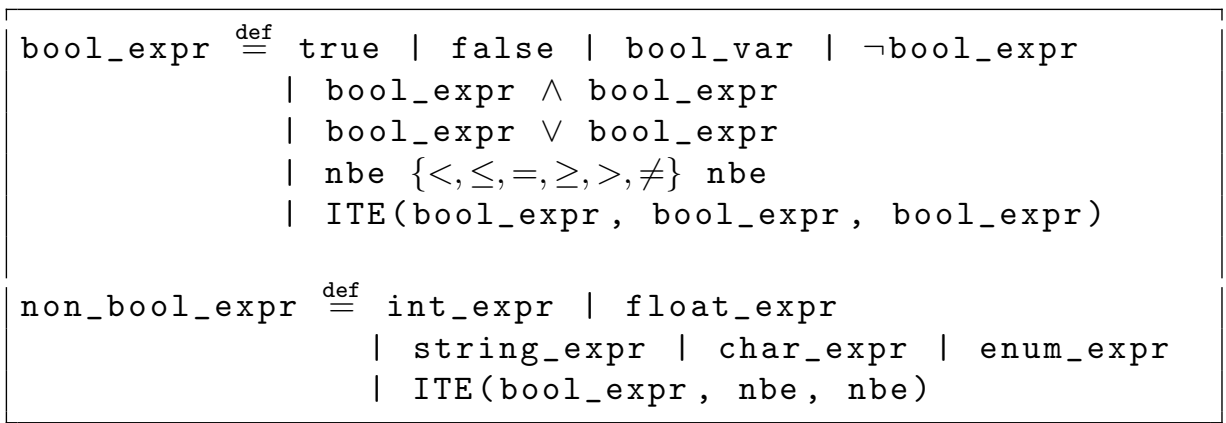

Listing 1: The mini syntax of a requirement

Most parts of the syntax are straightforward. The ITE is an if-then-else style operator like the ternary operator (? :) in C language, and nbe is short for non_bool_expr. This mini syntax does not aim to be comprehensive. For example, function/procedure definitions are not specified here. However, an argument to the function call in the form of the Boolean expression is treated as a bool_expr and a function return is regarded as a variable/expression of the corresponding type.

In addition, a Boolean predicate is defined as a Boolean expression of the form nbe $\sim$ nbe with $\sim \in\{<, \leq,=, \geq,>, \neq\}$. A Boolean predicate nbe $\sim$ nbe is also called an ordered predicate if $\sim \in\{<,=,>\}$. A compound Boolean expression is with multiple conjunctions or disjunctions of its Boolean sub-expressions.

\section{The Coverage Criterion}

We assume that the requirements are given as boo_expr as specified in Listing 1 . We now develop a coverage criterion in order to automatically apply a formalized procedure to each expression which generates a set of testing conditions that meaningfully interpret this requirement. The coverage criterion in our work extends $\mathrm{MC} / \mathrm{DC}$ with boundary value analysis (for compound expression analysis) to take control flow structures in the requirement specification into account.

\subsection{The compound expression}

Our treatment of compound expressions is similar to [13, which uses MC/DC together with boundary value analysis. However, we provide a formal definition of the rules for generating the testing conditions. 
In code coverage analysis, the $\mathrm{MC} / \mathrm{DC}$ criterion is often regarded as a good practice to generate testing conditions to examine a compound expression. According to $\mathrm{MC} / \mathrm{DC}$, the Boolean expression being tested is called the decision, which is going to be evaluated to true or false, and a condition of the decision is a Boolean predicate that cannot be broken down into simpler Boolean expressions. The MC/DC criterion requires that each condition needs to be evaluated one time to true and one time to false, and this flip of condition value affects the decision's output. For example, let us consider a requirement specified as in following compound expression.

$$
M \geq 0 \wedge N \leq 1000
$$

Applying the MC/DC rules, we obtain the testing conditions $\Phi=\{M \geq 0 \wedge N \leq$ $1000, \neg(M \geq 0) \wedge N \leq 1000, M \geq 0 \wedge \neg(N \leq 1000)\}$. More details on how to implement $\mathrm{MC} / \mathrm{DC}$ can be found in [14].

However, MC/DC is not immediately applicable for to software specifications, as it does not support equivalence class partitioning or boundary value analysis. Nevertheless, we believe MC/DC generates a meaningful set of testing conditions, which can be used as the starting point for adequately covering a requirement. Thus, subsequently to plain MC/DC, we perform two additional steps to obtain a larger set of testing conditions.

The motivation behind this is that an ordered predicate typically represents one equivalence class for the variables involved. As an example, given a nonordered predicate $M \geq 0$, by convention there are two equivalence classes: $M>0$ and $M=0$. In the following, we are going to introduce two operations that eliminate non-ordered predicates in any Boolean expression, by converting it into a set of Boolean expressions that do not contain non-ordered predicates.

Negation-free expressions A Boolean expression is said to be negation free for its predicates if there is no negation applied to any Boolean predicate within it. As an example, the two colored testing conditions in the set $\Phi$ above are not negation free.

Suppose that $e$ is a negated predicate of the form $e=\neg\left(n b e_{1} \sim n b e_{2}\right)$. We define a neg-free operator, as in Equation (2), to convert a negation expression to its equivalent set of non-negation expressions. By recursively applying it to any appearance of the negated predicate within an expression, the neg-free operator can be naturally extended to apply to any Boolean expression.

$$
n e g-f r e e(e)=\left\{\begin{aligned}
\left\{n b e_{1}=n b e_{2}, n b e_{1}>n b e_{2}\right\} & \text { if } \sim \text { is }< \\
\left\{n b e_{1}>n b e_{2}\right\} & \text { if } \sim \text { is } \leq \\
\left\{n b e_{1}<n b e_{2}, n b e_{1}>n b e_{2}\right\} & \text { if } \sim \text { is }= \\
\left\{n b e_{1}<n b e_{2}\right\} & \text { if } \sim \text { is } \geq \\
\left\{n b e_{1}=n b e_{2}, n b e_{1}<n b e_{2}\right\} & \text { if } \sim \text { is }> \\
\left\{n b e_{1}=n b e_{2}\right\} & \text { if } \sim \text { is } \neq
\end{aligned}\right.
$$


For the aforementioned example in (1), if we apply the negation free operator to its $\mathrm{MC} / \mathrm{DC}$ results, the resulting testing conditions are $\Phi=\{M \geq 0 \wedge N \leq$ $1000, M<0 \wedge N \leq 1000, M \geq 0 \wedge N>1000\}$. The colored predicates are due to neg-free.

Non-ordered predicate expansion Given a non-ordered Boolean predicate $e=n b e_{1} \sim n b e_{2}$ with $\sim \in\{\leq, \geq, \neq\}$, the to-ordered operator is defined to expand it into the equivalent ordered predicates.

$$
\text { to-ordered }(e)=\left\{\begin{array}{l}
\left\{n b e_{1}<n b e_{2}, n b e_{1}=n b e_{2}\right\} \text { if } \sim \text { is } \leq \\
\left\{n b e_{1}>n b e_{2}, n b e_{1}=n b e_{2}\right\} \text { if } \sim \text { is } \geq \\
\left\{n b e_{1}<n b e_{2}, n b e_{1}>n b e_{2}\right\} \text { if } \sim \text { is } \neq
\end{array}\right.
$$

Accordingly, for any testing condition generated after the MC/DC starting point, by conducting the neg-free and to-ordered operations, we obtain an elaborated set of testing conditions that respect equivalence class partitioning. As a result, for the example in (1), the final set of testing conditions will be $\Phi=\{M=0 \wedge N=1000, M=0 \wedge N<1000, M>0 \wedge N=1000$, $M>0 \wedge N<1000, M<0 \wedge N=1000, M<0 \wedge N<1000, M>0 \wedge N>1000$, $M=0 \wedge N>1000\}$. Clearly, the final results in $\Phi$ combine these equivalence classes for $M$ and $N$ based on their boundaries: in this case, $M=0$ and $N=1000$.

It is common to use a tolerance level $\sigma$ for boundary value analysis. The value of this tolerance level is based on the precision of the number representation and requirements for the accuracy of calculation. This combines well with our coverage criterion. In order to integrate this tolerance level, we extend the neg-free in (2) and to-ordered in (3) such that every time a testing condition with a predicate of the form $n b e_{1}<n b e_{2}$ (resp. nbe $\left.e_{1}>n b e_{2}\right)$ is obtained, an extra testing condition with this predicate replaced by $n b e_{1}=n b e_{2}-\sigma$ (resp. $\left.n b e_{1}=n b e_{2}+\sigma\right)$ is added to the set.

\subsection{The ITE expression}

So far, the proposed coverage criterion extends the MC/DC rules with the boundary value analysis, but it has no consideration of the possible control flow structure within a requirement, which is tackled in this part. The focus is on the (nested) if-then-else style structures encoded in the requirement. Each if-then-else (ITE) expression is represented as the tuple $\operatorname{ITE}\left(e_{1}, e_{2}, e_{3}\right)$, where $e_{1}, e_{2}$ and $e_{3}$ are all Boolean expressions such that $e_{2}$ will be reached only if $e_{1}$ is true, otherwise $e_{3}$ is chosen. Note that if $e_{2}$ and $e_{3}$ are not Boolean, the coverage analysis would stop at $e_{1}$. An example of the ITE expression is

$$
\operatorname{ITE}(M+N \leq 10, \text { Res }=M+N, \text { Res }=10) .
$$

Given any Boolean expression $e$, we use $\Phi(e)$ to denote its set of testing conditions after neg-free and to-ordered operations, following the MC/DC 
phase. Besides, $\Phi(e,+)$ contains all these testing conditions representing that the decision of $e$ is true and $\Phi(e,-)$ is for $e$ to be false such that $\Phi(e)=$ $\Phi(e,+) \cup \Phi(e,-)$.

Regarding any if-then-else expression $e=\operatorname{ITE}\left(e_{1}, e_{2}, e_{3}\right)$, the coverage analysis of $e_{2}$ (resp. $e_{3}$ ) will be only relevant if $e_{1}$ is true (resp. false). Therefore, we define the resultant testing conditions from an ITE expression $e$ as follows.

$$
\begin{aligned}
\Phi(e)= & \left\{e^{\prime} \mid \exists x \in \Phi\left(e_{1},+\right) \exists y \in \Phi\left(e_{2}\right) \text { s.t. } e^{\prime}=x \wedge y\right\} \\
& \cup\left\{e^{\prime} \mid \exists x \in \Phi\left(e_{1},-\right) \exists y \in \Phi\left(e_{3}\right) \text { s.t. } e^{\prime}=x \wedge y\right\}
\end{aligned}
$$

If we apply the definition in (5) to the ITE expression in (4), the final set of testing conditions generated becomes

$$
\begin{aligned}
\Phi=\{ & M+N<10 \wedge \text { Res }=M+N, \quad M+N<10 \wedge \\
& \operatorname{Res}>M+N, M+N<10 \wedge \text { Res }<M+N, \\
& M+N=10 \wedge \text { Res }=M+N, \quad M+N=10 \wedge \\
& \operatorname{Res}>M+N, M+N=10 \wedge \text { Res }<M+N, \\
& M+N>10 \wedge \text { Res }=10, \quad M+N>10 \wedge \text { Res } \\
& >10, \quad M+N>10 \wedge \text { Res }<10\} .
\end{aligned}
$$

It can be easily seen that if (4) is some requirement that must be guaranteed in the program, these colored testing conditions shall never be met.

\section{The Automated Testing Procedure}

In this section, we give the procedure for automated test generation from a function's requirements, which are specified as pre-conditions (Pre) and postconditions (Post). This is a generic framework to apply Bounded Model Checking (BMC) for automatically creating test cases from a function's requirements, and it relies on several routine methods (e.g., non-deterministic assignment) that are commonly available in modern Model Checkers. In our particular case, we implement this automated testing inside CBMC [8].

Suppose that these requirements are for a function/procedure $\mathcal{F}$, which has a set of input parameters $\mathcal{I}$ and a set of output parameters $\mathcal{O}$. The function $\mathcal{F}$ may also require some global input variables in $\mathcal{I}^{g}$ and after the function call it can change the values of some global output variables in $\mathcal{O}^{g}$.

In order to test the requirements of the target function $\mathcal{F}$, we require a calling context $\mathcal{F}^{c}$ for it. The calling context can be automatically generated, e.g., by a Python script. A concrete example is available in the appendix.

The calling context function $\mathcal{F}^{c}$ is structured as in Listing 2, REQUIRE is supposed to be the method that is supplied by a Model Checker. It takes a Boolean expression as the input argument and guarantees this expression is true/valid for the rest part of the program. It can also take string information as the second input argument, which is necessary for the traceability of tests, however, for simplicity this is not explicitly specified here. 


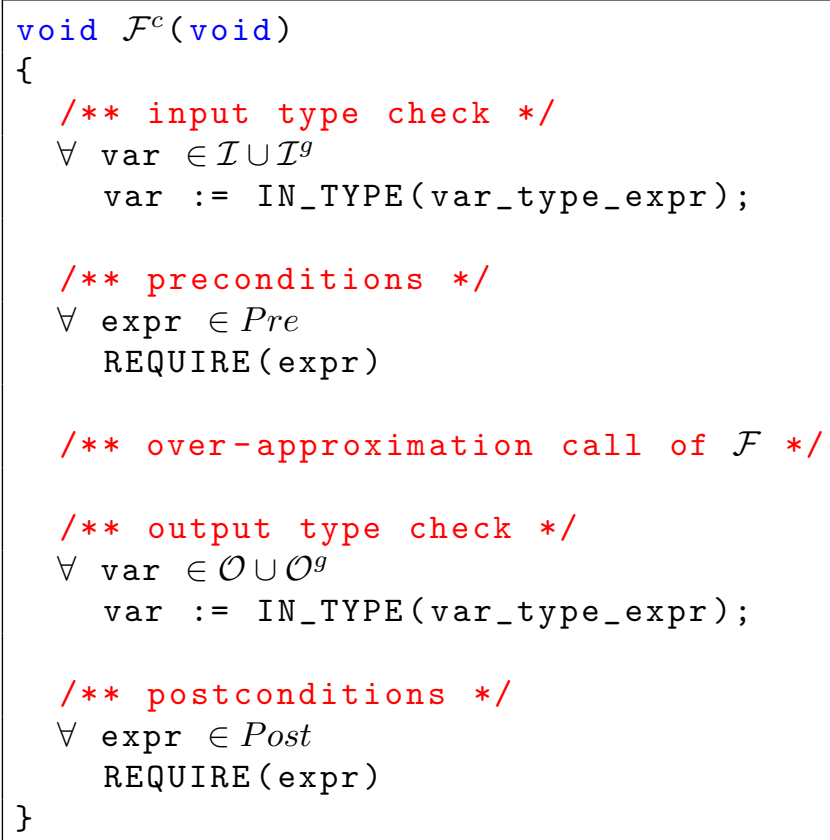

Listing 2: The calling context function

Initially, all inputs of the function $\mathcal{F}$ are non-deterministically assigned a value within the valid range of its type, by the IN_TYPE method, which contains two steps:

1. to generate a non-deterministic value for var;

2. to guarantee the value at step (1) is valid subject to var_type_expr and this is achieved by calling the REQUIRE method.

For any variable var, the var_type_expr is a Boolean expression depends on the type of var. For example,

- if var is a float variable, then var_type_expr is in form of min_float $\leq$ var $\leq$ max_float;

- if var is of an enum type, then var_type_expr is in form of var $=e^{\text {enum }} \mathrm{V} \vee$ var $=$ enum $_{2} \vee \ldots$, and it enumerates all possible values of var.

As a matter of fact, these input checks cope with the implicit requirements of software. That is, every input/output variable in the program inherently is constrained by its type.

Subsequently, for every requirement in the pre-conditions (Pre), a call to the REQUIRE method guarantees that it must be true for the following part of the program context. Because we aim to independently examine the requirements specified as pre-/post-conditions, the function body of $\mathcal{F}$ is simply ignored, 
whereas a proper over-approximation is also allowed depending on the application scenario. In consequence, outputs from $\mathcal{F}$ are over-approximately assigned values according to their types. To keep the consistency, requirements from the postconditions must be required to be true too, which indicates that our method does not target these conditions out of the range of the requirements.

In order to conduct the automated testing procedure, coverage criterion in Section 4 is supposed to have been already implemented in a Model Checker that is CBMC in this work. There are a number of works that implement or integrate different kinds of coverage analyses with Bounded Model Checking. Examples are like [15], [16] and [17. We have adopted a similar approach that embeds trap properties (i.e., negation of testing conditions) into the program model of these requirements.

The Model Checker simply goes through the calling context function $\mathcal{F}^{c}$ as listed and generates the set of testing conditions $\Phi$ (e.g., Equation (60) for every expr and var_type_expr, according to the coverage criterion defined.

For each testing condition (let us say) $\phi \in \Phi$, a trap property (that violates this testing condition) is encoded as $\neg \phi$ and instrumented into the $\mathcal{F}^{c}$ context, after which the Model Checker checks if these trap properties are satisfied.

- In case a (trap) property is not satisfied, then an execution trace of the program model (of $\mathcal{F}^{c}$ ) leading to the reachability of the violation of the property (i.e., the original testing condition) is returned and a test vector is outputted.

- Otherwise, it means that the corresponding testing condition will never be met.

In conclusion, a test vector in the results represents an admissible behaviour with respect to the function requirements, and it is expected that given the inputs as in a test vector, the execution of the function implementation shall trigger the corresponding testing conditions for examining the requirements, but it is not necessarily true that the exact values of outputs must be always the same, depending on the implementation choices.

\section{The Case Study}

The functional requirements-based test-generation method introduced in this paper has been implemented in the Model Checking tool CBMC, and is integrated into the toolchain developed in the AUTOSAC (Automated Testing of SPARK Ada Contracts) project.

The AUTOSAC toolchain is encapsulated within RapiTestFramework, the unit- and system-level testing solution provided by Rapita Systems. It serves as the graphical environment presented to the user as well as the solution for compiling, executing, and reviewing the generated tests. This toolchain's performance and ability to replace manual testing effort is confirmed through the process of generating and executing tests for selected code made available by Rolls-Royce. 


\subsection{Test compilation, execution and review}

As depicted in Figure 1. DO-178C certification requests evidence from both requirements coverage analysis and structural coverage analysis. This means, beyond automatically generating tests from requirements, a successful testing toolchain also needs to provide the following:

- executes the procedure or function under test with the test vector inputs;

- collects coverage data for execution of the subprogram, including statement, branch, and/or MC/DC coverage information;

- verifies that the Post-condition(s) for the subprogram have not been violated when the subprogram exits.

In addition, the data resulting from one or more test runs must be analyzed, collated, and presented to the tester in a format suitable for review. In this context, review may include either checking the successful execution of the test $(\mathrm{s})$ or reviewing the percentage of code coverage acheived during execution.

RapiTestFramework provides an existing solution for unit test execution and code coverage reporting, and has been extended in the following ways to acheive to achieve these goals:

- a dialog is presented to the tester displaying available subprograms for testing;

- a documented XML schema for test vector definitions has been exposed in order to allow communication with external tools such as CBMC;

- the generated test harness includes exception-handling for the purpose of reporting violated post-conditions.

The coverage reporting features of RapiTestFramework have been found to be suitable for review of test execution, especially code coverage percentages and source code highlighting and annotation.

\subsection{Overview of toolchain workflow}

The AUTOSAC case study required the integration of CBMC for test vector generation and RapiTestFramework for subprogram selection, test compilation and execution, and review of results.

This process is managed using a graphical environment to call the underlying tools. A brief explanation of each stage in the testing workflow follows, beginning with software requirements and concluding with test execution results.

The starting point for test generation is the low-level requirements of the functions and procedures under test, formulated as SPARK pre- and postconditions. Because the generated tests are based on these requirements, they have the same level of independence from software implementation as with a manual test-writing process. 
Firstly, these requirements are analyzed (by CBMC). Pre-/post-conditions and ranges of input/output parameter types define the scope of possible test vectors, and they are analyzed using strategies discussed in this paper for the test generation. To the extent to which these algorithms are successful, the collection of test vectors will be of similar quantity and quality to those that might have been written by a test engineer. The resulting test vectors are stored in a documented XML format.

Some configurable options were explored during the course of the case study which affect the resultant generated vectors, including limiting the unwinding of loops in the analysis of SPARK contracts, and the exploration of different combination levels of in-typed parameters for the coverage analysis, as in Figure 2 .

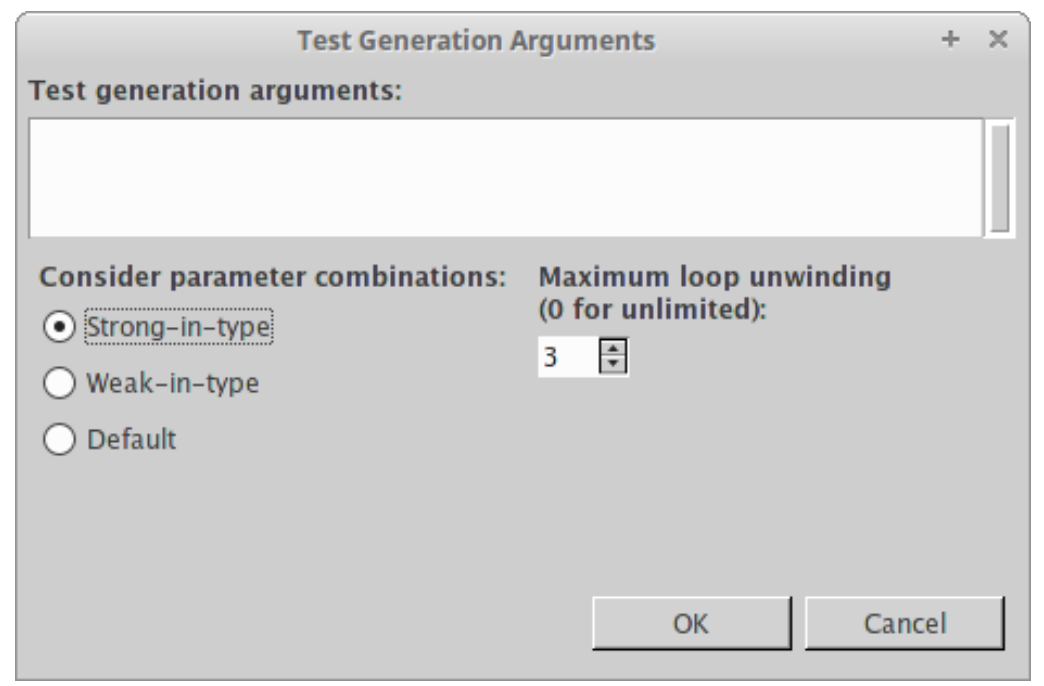

Figure 2: Toolchain configuration options

The next stage of the process converts these test vectors into executable test scripts (as in Figure 3). The scripts are presented to the user in their test project and are the principle artifact of the generation process. These tests may be altered by hand in the future, or simply used for test review; to facilitate this, the interface provides a text editor with syntax highlighting and error checking. Note that the testing condition is specified as part of the description, which provides the traceability back to requirements.

Finally, the GUI interface enables the user to compile and run a test executable including some or all of the generated tests. Upon completion of the test execution a report is created, which contains both test and code coverage results for analysis and review. Users can view coverage metrics at the function, statement, or $\mathrm{MC} / \mathrm{DC}$ level, providing immediate feedback on both the effectiveness of test generation as well as the coverage of the source code achieved from requirements, as in Figure 4. The coverage reporting features of RapiTestFramework, especially 


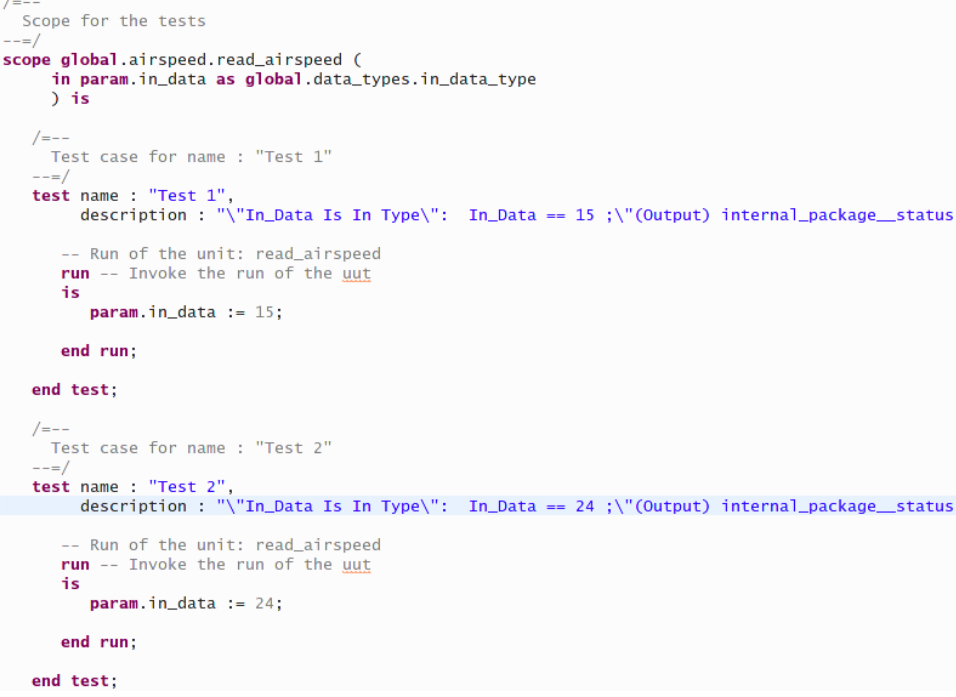

Figure 3: The test script

code coverage percentages and source code highlighting and annotation, have been found to be suitable for review of test execution.

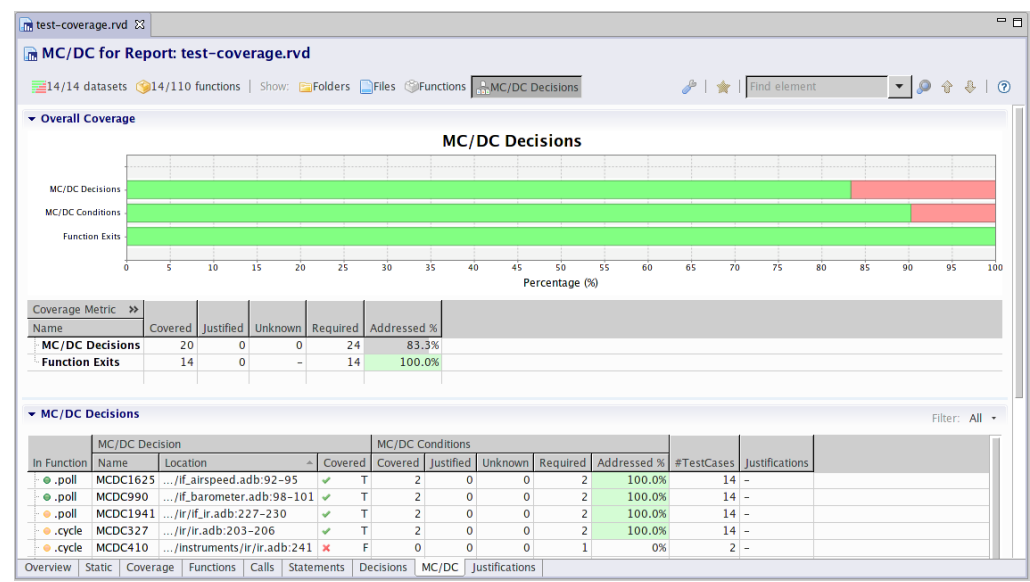

Figure 4: The code coverage analysis

In addition to the material presented here, a video demonstration of the toolchain (using open-source code under test) is available online ${ }^{1}$

${ }^{1}$ https://youtu.be/72NWFZQOvlM 


\subsection{Case study results}

The software architecture behind the case studies is composed of two layers. The first one is an application layer (AL) and the second one, is an operating layer $(\mathrm{OL})$. In order to use the toolchain in a real environment, two case studies have been picked, one per each layer in order to observe how the tool works with different abstract levels. Concretely, a health monitoring component for the AL case study and a temperature sensor component for the OL case study, both written in Ada, have been chosen.

After both components have been isolated from non-related code, to make the toolchain work, the legacy code has to be modified to represent the requirements with the component/functions by the use of SPARK Ada contracts, specifically, the use of SPARK pre- and post- conditions.

Ghost functions from SPARK2014 have not been used, as they are not currently supported by the toolchain. This has not been a problem for proceeding with the examples, though this would be a great advantage for the future.

By applying the AUTOSAC toolchain, tests are automatically generated from the SPARK Ada contracts. We check these resultant tests, by measuring the corresponding statement code coverage level on each subprogram: $A F A(100 \%)$, $A S T(100 \%), C T E 1(100 \%), C T E 2(100 \%), C T E C(100 \%), C V O(100 \%), F E S$ (100\%), ISCD(100\%), ISCF (100\%), ISCC(100\%), RTC $(68.75 \%)$.

Overall, the automatically generated tests show good coverage and represent a good spectrum of the case scenarios to be tested. The gap on code coverage level in some cases (e.g., $R T C$ from the OL) required further refinements on the SPARK Ada contracts under test and investigations on the intermediate language representation for SPARK Ada contracts in the toolchain.

Importantly, the verification role will retain its skillset, yet remove monotonous test scripting, as a result of writing SPARK Ada contracts instead of specific test harness. Besides writing such contracts, we believe minimal human observation for the review of the automatically generated tests is required.

The case study undertaken during the AUTOSAC research project demonstrated the successful implementation of automated testing in a real-world environment, integrating and extending existing tools for achieving DO-178-level certification. The toolchain has become one of the candidates for Rolls-Royce to reduce costs for verification, which is of great interest. We anticipate that it will undergo further development, including adding support for mixed-language test projects.

\subsection{Experience and lessons learned}

The main obstacle we encountered during the toolchain development phase is the intermediate representation of SPARK Ada. Regarding the automated testing procedure proposed in this paper, its implementation is based on CBMC, which does not have a direct front end for Ada. Thus, these requirements specified as in SPARK Ada contracts are at first converted into C language form, from which tests are then automatically generated. This choice is due to the limited 
timescale of the AUTOSAC project and is also for the purpose of evaluating the feasibility of such a requirements-based automated testing methodology. However, certain features in Ada cannot be trivially represented in $\mathrm{C}$ and the conversion procedure from Ada to $\mathrm{C}$ needs to be very carefully maintained. Currently, there is a continuing collaboration between partners to develop a formal Ada/SPARK front end for CBMC, thanks to the promising results shown from AUTOSAC toolchain for the automated test generation from software requirements.

Notwithstanding this limitation, the case study presented here illustrates the success of the tools and approach developed during the AUTOSAC project. In particular, the successful integration of CBMC and RapiTestFramework demonstrated the powerful combination of test generation based on mathematical proof combined with a robust platform for test execution and review. It is expected the reduction in time and cost of low-level requirement validation described in this paper will begin to be achieved as this technology is adopted in large-scale commercial projects.

On the other hand, defining requirements in the SPARK language has shown four advantages. Firstly, the different abstract levels allow us to generate low level tests, but also high level tests. Secondly, SPARK contracts provide an additional implicit verification between requirements and code design. Thirdly, it allows legacy code to become closer to new formal methods, as the evolution of SPARK language, so in future, the change would not be very radical. Finally, the use of the AUTOSAC toolchain encourages software teams to adopt agile development practices, as quick tests of the code can be done while maintaining independence from implementation, something that is currently not possible within the constraints of the DO-178 guidance.

Regarding this last point, such an agile approach allows the same person to perform two roles on different modules. The first one is the verification role, which would initially make the specification of some modules using SPARK Ada contracts. The other one is the design role, which would design/implement a different software module, independently from the SPARK Ada contracts written by another person.

\section{Conclusions}

In this paper, we successfully demonstrated feasibility of automatic test case generation from functional requirements, targeting software testing in avionics. We define a requirements-based coverage criterion and formulate an automated procedure for functional requirements-based testing. We implemented our method and integrated it within a testing environment. The developed toolchain is applied to industrial case studies, and its applicability and usefulness are confirmed.

Regarding future exploitation, we are interested in investigating application of the technique in this paper to test case chains generation for reactive systems [18. In particular, testing of model-based development processes (e.g., testing Simulink systems [19, 20, 21]) is of great relevance to the context of avionics 
software certification.

\section{Acknowledgement}

The work presented in this paper was sponsored by Birmingham City Council and Finance Birmingham and supported by Farnborough Aerospace Consortium (FAC) under NATEP project FAC043 - Automated Testing of SPARK Ada Contracts (AUTOSAC).

\section{References}

[1] RTCA, "DO-178C, Software considerations in airborne systems and equipment certification," 2011.

[2] S. C. Reid, "BS 7925-2: The software component testing standard," in Quality Software. Proceedings. First Asia-Pacific Conference on. IEEE, 2000, pp. 139-148.

[3] A. Gargantini and C. Heitmeyer, "Using model checking to generate tests from requirements specifications," in Software Engineering-ESEC/FSE. Springer, 1999, pp. 146-162.

[4] J. Chang and D. J. Richardson, "Structural specification-based testing: automated support and experimental evaluation," in Software Engineering-ESEC/FSE. Springer, 1999, pp. 285-302.

[5] R. M. Hierons, K. Bogdanov, J. P. Bowen, R. Cleaveland, J. Derrick, J. Dick, M. Gheorghe, M. Harman, K. Kapoor, P. Krause et al., "Using formal specifications to support testing," ACM Computing Surveys (CSUR), vol. 41, no. 2, p. 9, 2009.

[6] L. Tan, O. Sokolsky, and I. Lee, "Specification-based testing with linear temporal logic," in Information Reuse and Integration. Proceedings of the IEEE International Conference on, 2004, pp. 493-498.

[7] M. W. Whalen, A. Rajan, M. P. Heimdahl, and S. P. Miller, "Coverage metrics for requirements-based testing," in Proceedings of the international symposium on Software testing and analysis. ACM, 2006, pp. 25-36.

[8] E. Clarke, D. Kroening, and F. Lerda, "A tool for checking ANSI-C programs," in International Conference on Tools and Algorithms for the Construction and Analysis of Systems. Springer, 2004, pp. 168-176.

[9] RTCA, "DO-333, Formal methods supplement to DO-178C and DO-278A," 2011.

[10] M. Delahaye, N. Kosmatov, and J. Signoles, "Common specification language for static and dynamic analysis of C programs," in Proceedings of the 28th Annual ACM Symposium on Applied Computing. ACM, 2013, pp. 12301235 . 
[11] M. Barnett, K. R. M. Leino, and W. Schulte, "The Spec\# programming system: an overview," in International Workshop on Construction and Analysis of Safe, Secure, and Interoperable Smart Devices. Springer, 2004, pp. 49-69.

[12] B. John, "SPARK: The proven approach to high integrity software," 2012.

[13] J. J. Chilenski, "An investigation of three forms of the modified condition decision coverage (MCDC) criterion," DTIC Document, Tech. Rep., 2001.

[14] K. J. Hayhurst, D. S. Veerhusen, J. J. Chilenski, and L. K. Rierson, "A practical tutorial on modified condition/decision coverage," 2001.

[15] T. Ball, A Theory of Predicate-Complete Test Coverage and Generation. Berlin, Heidelberg: Springer Berlin Heidelberg, 2005, pp. 1-22.

[16] D. Angeletti, E. Giunchiglia, M. Narizzano, A. Puddu, and S. Sabina, "Using bounded model checking for coverage analysis of safety-critical software in an industrial setting," J. Autom. Reasoning, vol. 45, no. 4, pp. 397-414, 2010.

[17] P. V. Suman, T. Muske, P. Bokil, U. Shrotri, and R. Venkatesh, "Masking boundary value coverage: effectiveness and efficiency," in Testing-Practice and Research Techniques. Springer, 2010, pp. 8-22.

[18] P. Schrammel, T. Melham, and D. Kroening, "Generating test case chains for reactive systems," Software Tools for Technology Transfer (STTT), vol. 18, pp. 319-334, June 2016.

[19] A. Brillout, N. He, M. Mazzucchi, D. Kroening, M. Purandare, P. Rümmer, and G. Weissenbacher, "Mutation-based test case generation for Simulink models," in Formal Methods for Components and Objects (FMCO) 2009, ser. LNCS, vol. 6286. Springer, 2010, pp. 208-227.

[20] N. He, P. Rümmer, and D. Kroening, "Test-case generation for embedded Simulink via formal concept analysis," in Design Automation Conference (DAC). ACM, 2011, pp. 224-229.

[21] A. Balsini, M. Di Natale, M. Celia, and V. Tsachouridis, "Generation of Simulink monitors for control applications from formal requirements," in Industrial Embedded Systems (SIES), 12th IEEE Symposium on, 2017. 


\section{Appendix}

This is an illustrative example for the calling context function presented in Section 5. Given a target function $\mathcal{F}$ named as constrained_add, there are two input arguments $M$ and $N$, and one output Res. The function's requirements are specified as

$$
\begin{gathered}
\{M \geq 0 \wedge M \leq 10, \quad N \geq 0 \wedge N \leq 10, \quad \text { Res } \geq 0 \wedge \text { Res } \leq \\
10, \quad \operatorname{ITE}(M+N \leq 10, \text { Res }=M+N, \text { Res }=10)\}
\end{gathered}
$$

Using the AUTOSAC toolchain, the calling context function $\mathcal{F}^{c}$ is generated as in the code List 3 The two steps of IN_TYPE method in Section 5 correspond to the assignment of nondet__my_range and the call to __AUTOSAC_in_type.

As mentioned in Section 5 annotations can be also added as strings. For example, "postcondition at basic_tests.ads:52:20" tells that this is a post-condition that can be traced back to line 52 at the source file basic_tests.ads.

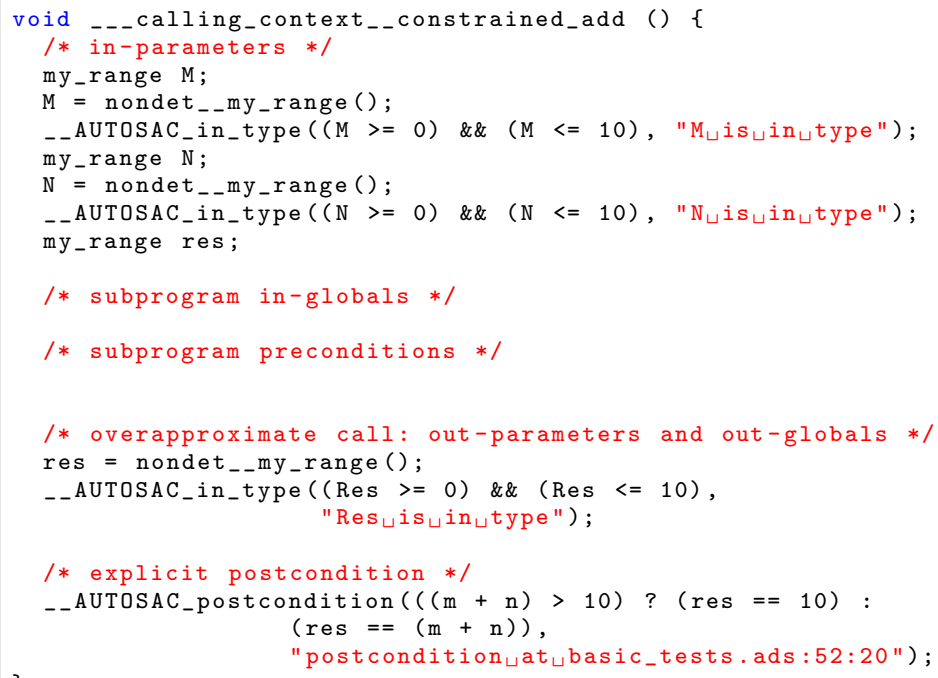

Listing 3: The calling context function for constrained_add

A calling context function in such a form can be now directly recognized and run through by CBMC. A set of testing conditions for each requirement of the constrained_add method will be generated according to the criterion in Section 4 . As an example, for the post-condition $\operatorname{ITE}(M+N \leq 10$, Res $=M+N$, Res = 10), the set of testing conditions is as in (6). Afterwards, these conditions are negated and instrumented by CBMC to automatically generate test cases. The whole procedure of test generation is independent from the implementation of constrained_add function. 